\title{
Analyzing transfer learning impact in biomedical cross-lingual named entity recognition and normalization
}

\author{
Renzo M. Rivera-Zavala* (10) and Paloma Martínez
}

From The 5th workshop on BioNLP Open Shared Tasks Hong Kong, China. 4 November 2019

*Correspondence:

renzomauricio.

rivera@alumnos.uc3m.es

Computer Science

Department, University

Carlos III of Madrid, Leganes,

Madrid, Spain

\begin{abstract}
Background: The volume of biomedical literature and clinical data is growing at an exponential rate. Therefore, efficient access to data described in unstructured biomedical texts is a crucial task for the biomedical industry and research. Named Entity Recognition (NER) is the first step for information and knowledge acquisition when we deal with unstructured texts. Recent NER approaches use contextualized word representations as input for a downstream classification task. However, distributed word vectors (embeddings) are very limited in Spanish and even more for the biomedical domain.
\end{abstract}

Methods: In this work, we develop several biomedical Spanish word representations, and we introduce two Deep Learning approaches for pharmaceutical, chemical, and other biomedical entities recognition in Spanish clinical case texts and biomedical texts, one based on a Bi-STM-CRF model and the other on a BERT-based architecture.

Results: Several Spanish biomedical embeddigns together with the two deep learning models were evaluated on the PharmaCoNER and CORD-19 datasets. The PharmaCoNER dataset is composed of a set of Spanish clinical cases annotated with drugs, chemical compounds and pharmacological substances; our extended Bi-LSTMCRF model obtains an F-score of $85.24 \%$ on entity identification and classification and the BERT model obtains an F-score of $88.80 \%$. For the entity normalization task, the extended Bi-LSTM-CRF model achieves an F-score of $72.85 \%$ and the BERT model achieves 79.97\%. The CORD-19 dataset consists of scholarly articles written in English annotated with biomedical concepts such as disorder, species, chemical or drugs, gene and protein, enzyme and anatomy. Bi-LSTM-CRF model and BERT model obtain an F-measure of $78.23 \%$ and $78.86 \%$ on entity identification and classification, respectively on the CORD-19 dataset.

Conclusion: These results prove that deep learning models with in-domain knowledge learned from large-scale datasets highly improve named entity recognition performance. Moreover, contextualized representations help to understand complexities and ambiguity inherent to biomedical texts. Embeddings based on word, concepts, author(s) and the source, provide a link to the Creative Commons licence, and indicate if changes were made. The images or other third party material in this article are included in the article's Creative Commons licence, unless indicated otherwise in a credit line to the material. If material is not included in the article's Creative Commons licence and your intended use is not permitted by statutory regulation or exceeds the permitted use, you will need to obtain permission directly from the copyright holder. To view a copy of this licence, visit http:// creativecommons.org/licenses/by/4.0/. The Creative Commons Public Domain Dedication waiver (http://creativecommons.org/publi cdomain/zero/1.0/) applies to the data made available in this article, unless otherwise stated in a credit line to the data. 
senses, etc. other than those for English are required to improve NER tasks in other languages.

Keywords: Natural language processing, Clinical texts, Deep learning, Contextual information

\section{Background}

Efficient information extraction off biomedical data described in scientific articles, clinical narrative, or e-health reports is a growing interest in biomedical industry, research, and so forth. In this context, improved biomedical name mentions identification in the biomedical texts is a crucial step downstream tasks such as drug and protein interactions, chemical compounds, adverse drug reactions, among others. Named Entity Recognition (NER) is one of the fundamental tasks of biomedical text processing, intending to automatically extract and identify mentions of entities of interest in running text, typically through their mention boundary or by classifying tokens to match specific entity mentions. Traditionally, there are three phases in recognizing concepts in texts: (1) to identify the limits of the term or phrase that represents the concept in the text (char offsets in the text), (2) to classify the term or phrase on a class (for instance, drug, disease, body part, etc.) and (3) to normalize the concept by assigning it an identifier in a specific domain resource such as UMLS [1]. The existing biomedical NER methods can be classified into: dictionary-based methods, which are based on the use of existing domain knowledge dictionaries limited by its size, spelling errors, the use of synonyms, and the constant growth of vocabulary. Rule-based methods and Machine Learning methods usually depend on the engineering of syntactic and semantic features as well as specific language and domain features that are learned from large collections of text or built from scratch. More recently, deep learning approaches have emerged due to the availability of myriad data from different sources (scientific literature, social media, clinical texts, etc.).

The NER task has been accomplished by three types of methods. Dictionary-based methods require having specific resources integrating terminology such METAMAP tool [2] that includes UMLS [1] and recognizes mentions of medical concepts. With the availability of annotated corpora, machine learning supervised approaches have widely used in entity recognition. One of the most effective methods is Conditional Random Fields (CRF) [3] since CRF is one of the most reliable sequence labeling methods. Different challenges have been held to foster research in NER, for example, eHealth CLEF, SEMEVAL and TAC, among others. In the special case of drugs, DDIExtraction 2011 [4] and DDIExtraction 2013 [5] were specifically designed to recognize pharmacological entities and drug-drug interactions (DDI) in Medline abstracts and DrugBank technical records both in English. In these shared tasks the best result reported for NER using four types of pharmacological substances (generic drug names, branded drug names, drug group names and active substances not approved for human use) was F1 of 71.5\% (by a system based on CRF algorithm). For DDI identification and classification in four classes (advice, mechanism, effect and int) the best result was 65.1\% (system bases on a combination of kernels). Most of the participating systems were built on support vector machines (SVM). In general, approaches based on non-linear kernels methods achieved better results than linear SVMs. 
More recently, deep learning methods started to obtain better results in NER based on the use of pre-trained models (word embeddings) obtained from a huge volume of unlabelled texts (scientific literature, social media texts, Wikipedia, among others). Word embeddings have been evolving from static representations that do not model the dynamic nature of words to contextualized representations that allow word embeddings to adapt to the context it appears, see [6] for a detailed description of embeddings. Pretrained models may be useful for analyzing texts if these texts are similar to what they were trained on. When texts are from a different domain we will need to fine-tune a pre-trained model to fit our data or task. This is much more efficient than training a whole model from scratch because it is too time and resources consuming task. With a limited set of examples systems can get high performance in downstream tasks. See [7] for a survey of embeddings in clinical natural language processing. The new challenge, PharmacoNER 2019 [8] was focused on recognizing and normalizing pharmacological substances in Spanish clinical cases. In the current stream of deep learning approaches, participating systems mostly included those architectures. The baseline defined for PharmacoNER was based on vocabulary transfer using a LSTM model with Glove embeddings trained from SBWC and the medical word embeddings for Spanish [9] that achieved a high F1 of $0.82 \%$ (ranked 16 out of 22) in NER. The first ranked system [10] was based on a pipeline composed of a BERT (Bidirectional Encoder Representations from Transformers) for NER and a Bi-LSTM for concept indexing achieving an F1-score of $91.5 \%$ on NER and $83.9 \%$ on concept indexing. The third-ranked system [11] was based on a Bi-LSTM-CRF tagger with FLAIR contextualized embeddings obtaining a result of $89.76 \%$ F1-score using pre-trained embeddings and up to $90.5 \%$ using specialized ones. The second-ranked system [12] implemented a traditional knowledge-based approach based on dictionaries, particularly the SNOMED-CT medical ontology [13] together with a set of 104 contextual regexp patterns to tackle ambiguity (an important issue especially for abbreviations) and surprisingly this system obtained an F1-Score of 91\% in NER and 91.6\% in concept indexing (top system). This reveals that resource-based approaches have a lot to say yet. Other deep learning works have also demonstrated state-of-the-art performance for English [14-16] texts by automatically learning relevant patterns from corpora, which allows language and domain independence. Weber [17] described a set of experiments with a NER tool called HUNER that incorporates a fully trained LSTM-CRF model using 34 different corpora for five entity types that outperform the state-of-the-art tools CnormPlus and tmChem by $5-13 \mathrm{pp}$ for chemicals, species and gene on CRAFT corpus [18]. However, concerning the generation of domain-based pre-trained models until now, to the best of our knowledge, there is only one work that addresses the generation of Spanish biomedical word embeddings $[9,19]$.

In this paper, we propose two deep learning approaches to face the recognition of pharmacological and chemical entities in Spanish texts. The approaches are evaluated using the Spanish biomedical PharmaCoNER and English biomedical CORD-19 datasets. Our main goal is to evaluate the performance impact of cross-domain (general and biomedical domain) and cross-language (Spanish and English) pre-trained embeddings models. Firstly, for entity identification and classification, we implemented two bidirectional Long Short Memory (Bi-LSTM) layers with a CRF layer based on the NeuroNER 
model proposed in [20]. Specifically, we have extended the NeuroNER [20] architecture by adding context information to token-level representation, such as Part-of-Speech (PoS) tags and overlapping or nested entities. Moreover, in this work, we use several pretrained word embedding models: (i) a word2vec model (Spanish Billion Word Embeddings [21]), which was trained on the 2014 dump of Wikipedia, (ii) pre-trained word2vec model of word embeddings trained with PubMed and PMC articles, (iii) Scielo and Wikipedia cased pre-trained model based on the FastText implementation, (iv) a sensedisambiguation embedding model [22], where different word senses are represented with different sense vectors and trained from scratch embedding models (v) the FastText-SBC model trained on the FastText implementation and (vi) the SNOMED-SBC model based on the FastText-SBC replacing concepts with their unique SNOMED-CT [13] identifier. Finally, we implemented the Bidirectional Encoder Representations for Transformers (BERT) model with fine-tuning using BERT pre-trained general domain models and a trained from scratch biomedical model. For concept indexing based on the output of offset recognition and entity classification, we applied a full-text search and a fuzzy matching approach on the SNOMED-CT Spanish Edition dictionary to obtain the corresponding index to normalize the concept.

\section{Results}

We evaluate our deep learning models using the train, validation and test datasets provided by the task organizers of the PharmaCoNER Shared Task [8]. The PharmaCoNER task considers two subtasks. Subtask 1 considers offset recognition and entity classification of pharmacological substances, compounds, and proteins. Subtask 2 considers concept indexing where for each entity, the list of unique SNOMED concept identifiers must be generated. We apply the standard measures precision, recall and F1-score to evaluate the performance of our approaches. These metrics are also used in the PharmaCoNER task. A detailed description of the evaluation can be found in [23].

Moreover, we evaluate our deep learning models on the train, validation and test subsets of the CORD-19 dataset [24]. F-measure is used as the primary metric where true positives are entities that match with the gold standard annotations boundaries and entity type.

\section{Offset detection and entity classification}

The NER task is addressed as a sequence labeling task. For NER we tested different configurations with various pre-trained word representation models.

\section{Bi-LSTM CRF model: extended NeuroNER}

For our Bi-LSTM CRF model we test various pre-trained and trained from the scratch word embeddings models (see Table 21). Table 1 describes our different experiment configurations for the PharmaCoNER datasets with Spanish general domain (W2VSBWC and FastText-SBWC), English general domain (FastText 2M), Spanish biomedical domain (FastText-SBC and SNOMED-SBC) and English biomedical domain (PubdMed and PMC) embeddings. Each configuration for all evaluations was executed up to 5 times and we kept the best result obtained (85.75) as shown in Table 2. Table 2 compares 
Table 1 System hyperparameters for each PharmaCoNER run

\begin{tabular}{lllll}
\hline Parameter & Run 1 & Run 2 & Run 3 & Run 4 \\
\hline Sense-disambiguation embedding dimension & 128 & 128 & 128 & 128 \\
Pre-trained word embeddings & $\begin{array}{l}\text { FastText-SBC } \\
\text { + Reddit }\end{array}$ & $\begin{array}{l}\text { W2V-SBWC } \\
\text { Reddit }\end{array}$ & $\begin{array}{l}\text { FastText-SBWC } \\
\text { + Reddit }\end{array}$ & $\begin{array}{l}\text { SNOMED- } \\
\text { SBC + } \\
\text { Reddit }\end{array}$ \\
Word embeddings dimension & & & & 300 \\
Character embedding dimension & 300 & 300 & 300 & 50 \\
Hidden layers dimension (for each LSTM) & 50 & 50 & 50 & 100 \\
Learning method & 100 & 100 & 100 & SGD \\
Dropout rate & SGD & SGD & SGD & 0.5 \\
Learning rate & 0.5 & 0.5 & 0.5 & 0.005 \\
Epochs & 0.005 & 0.005 & 0.005 & 100 \\
\hline
\end{tabular}

Table 2 Extended NeuroNER with FastText-SBC + Reddit embedding models runs results according to Table 1 configurations

\begin{tabular}{llll}
\hline Experiment & Precision (\%) & Recall (\%) & F-score (\%) \\
\hline Run 1 & 88.19 & 82.61 & 85.31 \\
Run 2 & 87.77 & $\mathbf{8 3 . 6 5}$ & 85.66 \\
Run 3 & $\mathbf{9 0 . 0}$ & 80.9 & 85.21 \\
Run 4 & 89.13 & 82.61 & $\mathbf{8 5 . 7 5}$ \\
Run 5 & 88.03 & 82.96 & 85.42
\end{tabular}

Bold values are the best results for Precision (P), Recall (R) and F-score

Table 3 Extended NeuroNER results for each entity on PharmaCoNER valid dataset

\begin{tabular}{lccc}
\hline Entity & Precision (\%) & Recall (\%) & F-score (\%) \\
\hline Normalizables & 92.38 & $\mathbf{8 6 . 4 1}$ & $\mathbf{8 9 . 2 9}$ \\
No_Normalizables & 0.00 & 0.00 & 0.00 \\
Proteins & $\mathbf{9 3 . 2 9}$ & 85.35 & 89.14 \\
Unclear & 87.80 & 70.59 & 78.26 \\
Micro-average & 91.75 & 84.74 & 88.10 \\
\hline
\end{tabular}

Bold values are the best results for Precision (P), Recall (R) and F-score

the different results obtained in 5 runs for Extended NeuroNER using FastText-SBC + Reddit embedding models.

Table 4 shows a comparison of the different pre-trained models on the PharmaCoNER validation dataset where we want to highlight that domain-specific and word embeddings outperform general domain models by almost 5 points, Moreover, language-specific word embeddings outperform cross-lingual models by almost 4 points. Furthermore, lower performance of general domain and cross-lingual word embeddings models can be related to recall performance; this can be interpreted as many out-ofvocabulary words. For the test dataset, we applied our best system configuration FastText-SBC + Reddit (see Table 4) obtaining an F-score of $85.24 \%$ for offset detection and entity classification. Furthermore, Table 3 shows the classification results obtained by 
Table 4 Results for Extended NeuroNER entity classification using combinations of embeddings models on PharmaCoNER test dataset

\begin{tabular}{lllll}
\hline Experiment & Embedding model & Precision (\%) & Recall (\%) & F-score (\%) \\
\hline Run 4 & SNOMED-SBC + Reddit & 83.52 & 74.97 & 79.02 \\
Run 2 & W2V-SBWC + Reddit & 83.85 & 75.75 & 79.60 \\
Run 3 & FastText-SBWC + Reddit & 84.70 & 77.31 & 80.84 \\
Run 1 & FastText-SBC + Reddit & $\mathbf{8 9 . 1 3}$ & 82.61 & $\mathbf{8 5 . 7 5}$ \\
Out of task & Scielo+Wiki cased + Reddit & 86.69 & $\mathbf{8 2 . 7 2}$ & 84.66 \\
Out of task & PubMed and PMC + Reddit & 87.23 & 76.98 & 81.79 \\
Out of task & FastText 2M + Reddit & 84.04 & 77.55 & 80.67 \\
\hline
\end{tabular}

Bold values are the best results for Precision (P), Recall (R) and F-score

our best system configuration for offset detection and entity classification with a micro average of $88.10 \%$ for PharmaCoNER valid dataset.

Moreover, we compared our best system configuration (FastText-SBC + Reddit) with the baseline NeuroNER model (without sense embeddings and BMEWO-V format encoding) using the same FastText-SBC embedding and configuration. Table 5 shows that our extended system outperforms the NeuroNER base system, which has proven that sense embeddings and BMEWO- $\mathrm{V}$ format to be an additional source of information to deal with ambiguity and nested entities (see "Methods" section for detail about BMEWO-V format). Furthermore, the use of domain-specific word embeddings highly improves performance as is shown in Table 4.

Furthermore, we tested the FastText-2M English general domain and the Pubmed and PMC English domain-specific non-contextualized pre-trained embeddings models (more details in Table 21) on the CORD-19 dataset. Table 6 describes our different experiment configurations for the CORD-19 dataset.

Table 5 Baseline comparison for entity classification on PharmaCoNER test dataset

\begin{tabular}{llll}
\hline System & Precision (\%) & Recall (\%) & F-score (\%) \\
\hline NeuroNER & 86.38 & 82.07 & 84.16 \\
Extended NeuroNER & $\mathbf{8 9 . 1 3}$ & $\mathbf{8 2 . 6 1}$ & $\mathbf{8 5 . 7 5}$ \\
\hline
\end{tabular}

Bold values are the best results for Precision (P), Recall (R) and F-score

Table 6 System hyperparameters for CORD-19 experiments

\begin{tabular}{lll}
\hline Parameter & Experiment 1 & Experiment 2 \\
\hline Sense-disambiguation embedding dimension & 128 & 128 \\
Pre-trained word embeddings & Pubmed and PMC + Reddit & FastText 2M + Reddit \\
Word embeddings dimension & 300 & 300 \\
Character embedding dimension & 50 & 50 \\
Hidden layers dimension (for each LSTM) & 100 & 100 \\
Learning method & $\mathrm{SGD}$ & $\mathrm{SGD}$ \\
Dropout rate & 0.5 & 0.5 \\
Learning rate & 0.005 & 0.005 \\
Epochs & 100 & 100 \\
\hline
\end{tabular}


Table 7 Extended NeuroNER results for each entity on CORD-19 test dataset

\begin{tabular}{llll}
\hline Entity & Precision (\%) & Recall (\%) & F-score (\%) \\
\hline Chemical or Drug & 81.86 & $\mathbf{8 3 . 5 2}$ & 82.68 \\
Disorder & $\mathbf{8 5 . 7 3}$ & 80.77 & $\mathbf{8 3 . 1 7}$ \\
Protein or Gene & 63.81 & 49.40 & 55.69 \\
Micro-average & 81.17 & 75.49 & 78.23
\end{tabular}

Bold values are the best results for Precision (P), Recall $(\mathrm{R})$ and F-score

Table 8 Extended NeuroNER results for entity classification on CORD-19 test dataset

\begin{tabular}{llll}
\hline Embedding model & Precision (\%) & Recall (\%) & F-score (\%) \\
\hline Pubmed and PMC + Reddit & $\mathbf{8 1 . 1 7}$ & $\mathbf{7 5 . 4 9}$ & $\mathbf{7 8 . 2 3}$ \\
FastText 2M + Reddit & 77.77 & 73.71 & 75.69 \\
\hline
\end{tabular}

Bold values are the best results for Precision (P), Recall $(\mathrm{R})$ and F-score

Table 9 Results of BERT systems for entity classification on PharmaCoNER test dataset

\begin{tabular}{llll}
\hline System & Precision (\%) & Recall (\%) & F-score (\%) \\
\hline bert-base-multilingual-cased & 84.01 & 76.91 & 80.23 \\
BETO cased & 84.68 & 79.02 & 81.66 \\
SBC-BERT & $\mathbf{8 7 . 8 8}$ & $\mathbf{8 9 . 7 4}$ & $\mathbf{8 8 . 8 0}$ \\
\hline
\end{tabular}

Bold values are the best results for Precision (P), Recall (R) and F-score

In Table 8, we compare the FastText-2M model trained on English general domain texts and Pubmed and PMC model trained on English biomedical texts (more details in Table 22), both tested on the CORD-19 test dataset. As shown in Table 8, domainspecific models outperform general domain models by almost 3 points, obtaining an F-score of $78.23 \%$ for offset detection and entity classification. Table 7 shows the classification results obtained by our best system configuration for offset detection and entity classification with a micro average F-score of 78.23\% for the CORD-19 test dataset. Classification results on Protein/Gene are lower than other entities type mainly due to ambiguity and short named entity detection.

\section{Multi-layer bidirectional transformer encoder: BERT}

Additionally, we compare the different contextualized word models using the BERT implementation on the PharmaCoNER and CORD-19 test dataset with 12 transformer layers, 768-hidden, 12-heads, $110 \mathrm{M}$ parameters trained on each pre-trained model and fine-tuned for NER using a single output layer based on the representations from its last layer to compute only token level BIOES-V probabilities. BERT directly learns WordPiece embeddings during pre-training and fine-tuning steps. BERT provides subword representations. Subwords are used for representing both the input text and the output tokens. Out of vocabulary words are sliced into multiple subwords, even reaching character subwords if needed. However, subwords representations do not necessarily fit with word representation in a given context. 
Table 10 Results of SBC-BERT system for entity classification on test PharmaCoNER dataset

\begin{tabular}{llll}
\hline Entity & Precision (\%) & Recall (\%) & F-score (\%) \\
\hline PROTEINAS & 84.46 & 88.46 & 86.41 \\
NORMALIZABLES & $\mathbf{9 1 . 8 6}$ & $\mathbf{9 2 . 0 2}$ & $\mathbf{9 1 . 9 4}$ \\
UNCLEAR & 70.59 & 81.82 & 75.79 \\
NO_NORMALIZABLES & 15.38 & 12.5 & 13.79 \\
micro avg & 87.88 & 89.74 & 88.80 \\
\hline
\end{tabular}

Bold values are the best results for Precision (P), Recall $(\mathrm{R})$ and F-score

Table 11 Comparison of participant systems and ours on PharmaCoNER test dataset

\begin{tabular}{llll}
\hline Name & Precision (\%) & Recall (\%) & F-score (\%) \\
\hline xiongying [10] & $\mathbf{9 1 . 2 2}$ & 90.87 & $\mathbf{9 1 . 0 5}$ \\
FSL [12] & 90.62 & $\mathbf{9 1 . 3 1}$ & 90.96 \\
m-stoeckel [11] & 90.70 & 89.08 & 90.46 \\
CongSun [26] & 88.05 & 89.24 & 88.64 \\
SBC-BERT & 87.88 & 89.74 & 88.80 \\
Extended NeuroNER & 89.13 & 82.61 & 85.75
\end{tabular}

Bold values are the best results for Precision (P), Recall (R) and F-score

Table 12 BERT results for each entity on CORD-19 test dataset

\begin{tabular}{llll}
\hline Entity & Precision (\%) & Recall (\%) & F-score (\%) \\
\hline Chemical or Drug & $\mathbf{8 6 . 0 5}$ & 83.71 & $\mathbf{8 4 . 8 6}$ \\
Disorder & 83.68 & $\mathbf{8 4 . 3 7}$ & 84.02 \\
Protein or Gene & 54.00 & 65.06 & 59.02 \\
Micro-average & 77.28 & 80.52 & 78.86 \\
\hline
\end{tabular}

Bold values are the best results for Precision (P), Recall (R) and F-score

Table 13 Contextualized word models results for entity classification on CORD-19 test dataset

\begin{tabular}{llll}
\hline System & Precision (\%) & Recall (\%) & F-score (\%) \\
\hline bert-base-multilingual-cased & 72.12 & 75.92 & 73.89 \\
BioBERT Large & $\mathbf{7 7 . 2 8}$ & $\mathbf{8 0 . 5 2}$ & $\mathbf{7 8 . 8 6}$ \\
\hline
\end{tabular}

Bold values are the best results for Precision (P), Recall (R) and F-score

We compare the different general domain English pre-trained (bert-base-multilingualcased and BETO cased) and domain-specific English pre-trained (SBC-BERT) contextualized word embeddings. As shown in Table 9 domain-specific word representations outperform general domain models by almost 7 points. Nonetheless, to the best of our knowledge there is no open pre-trained contextualized word biomedical Spanish model. Moreover, Table 10 shows the classification results obtained by our best model for offset detection and entity classification with a micro average F-score of $88.80 \%$ for PharmaCoNER test dataset.

We compare our deep learning approaches with the participating systems presented in the PharmaCoNER task. A detailed description of the evaluation and the participant systems is provided in [25]. As can be seen in Table 11, our SBC-BERT model reaches 
satisfactory performance, however is outperformed by other approaches adding more complex language and domain-specific features.

Moreover, we test different contextualized word pre-trained models on the CORD-19 test dataset. As shown in Table 13, domain-specific word representations outperform general domain models by almost 5 points. Based in our experiments, we found that the use of domain-specific contextualized word representations highly improves the entity classification task. Table 12 shows the classification results obtained by our best BERT system configuration for offset detection and entity classification with a micro average of 78.86\% for CORD-19 test dataset.

\section{Concept indexing}

For concept indexing or normalization, we applied the same approach described for SNOMED-SBC model training, replacing each entity detected in the entity recognition and classification step with their unique SNOMED-CT Spanish Edition identifier. First, we applied a lowercase conversion, then we replace abbreviations with their corresponding full concept name using the Spanish Medical Abbreviation DataBase (AbreMES-DB) [27] and the SEDOM Medical Abbreviation Dictionary [28] for normalizing biomedical entities. We used the PyMedTermino library employing a two-stage search using full-text search and fuzzy search for concepts not found by partial matching. A full-text search with the Levenshtein distance algorithm [29] was applied in a first instance for concept indexing and fuzzy search with threshold using FuzzyDict implementation [14] as a second approach for concepts not found in the first instance by partial matching. Table 14 shows our result on concept indexing for PharmaCoNER test subset. We apply the standard measures precision, recall and micro-averaged F1-score to evaluate the effectiveness of our model, given as the evaluation metrics by the PharmaCoNER NER and concept indexing task. Results from the previous NER step are passed over for concept indexing. As shown in Table 14, BERT approach outperforms Extended NeuroNER mainly for the ability of BERT approach to resolve ambiguity.

Our results for concept indexing are low due to a large number of misspellings entities, abbreviations ambiguity, drug names where the identifier corresponds to the active substance as "durogesic" ("Duragesic") active ingredient "fentanyl" ("fentanyl"), identifiers not existing in SNOMED CT, such as CHEBI:135810 and 373757009 and false positives, such as diseases identified as NORMALIZABLE entities and PROTEIN entities not annotated in the PharmaCoNER corpus.

\section{Discussion}

We used different pre-trained models and investigated their effect on performance. For Extended NeuroNER, we used general and specific-domain pre-trained word embedding models, likewise we used pre-trained multi-language and language-specific models.

Table 14 Results for concept indexing on PharmaCoNER test dataset

\begin{tabular}{llll}
\hline System & Precision (\%) & Recall (\%) & F-score (\%) \\
\hline SBC-BERT & $\mathbf{8 7 . 3 4}$ & $\mathbf{7 3 . 7 5}$ & $\mathbf{7 9 . 9 7}$ \\
Extended NeuroNER & 84.17 & 64.22 & 72.85 \\
\hline
\end{tabular}

Bold values are the best results for Precision (P), Recall (R) and F-score 
Table 15 PharmaCoNER confusion matrix on test dataset for Extended NeuroNER best configuration

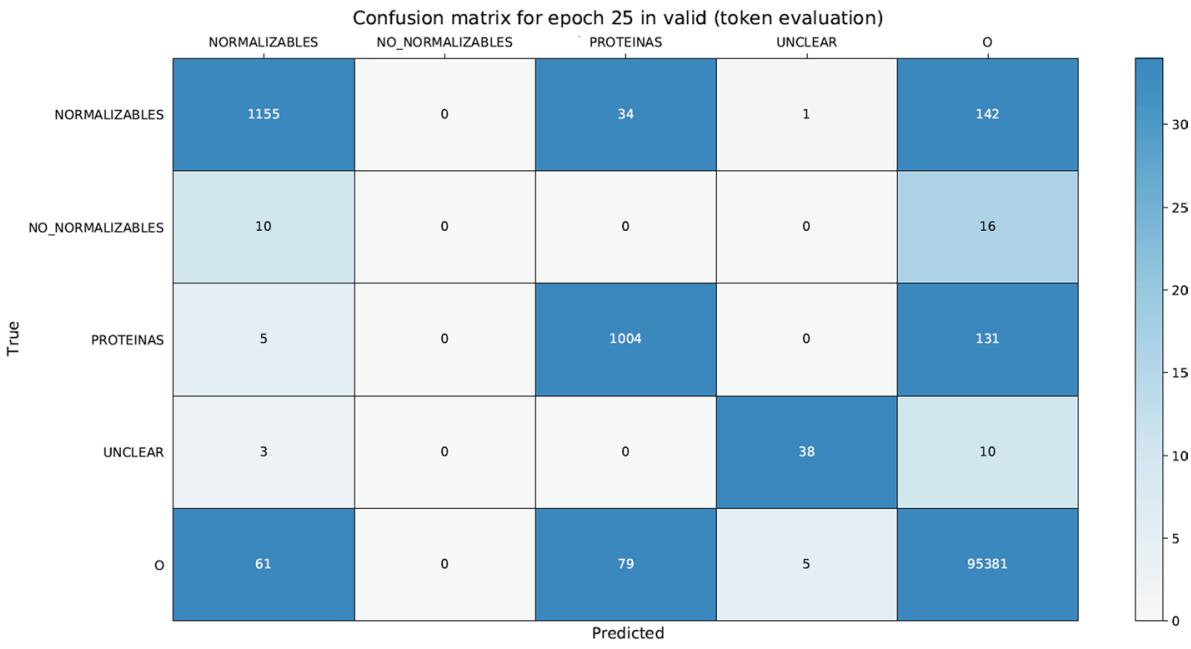

Table 16 CORD-19 confusion matrix on valid dataset for Extended NeuroNER best configuration

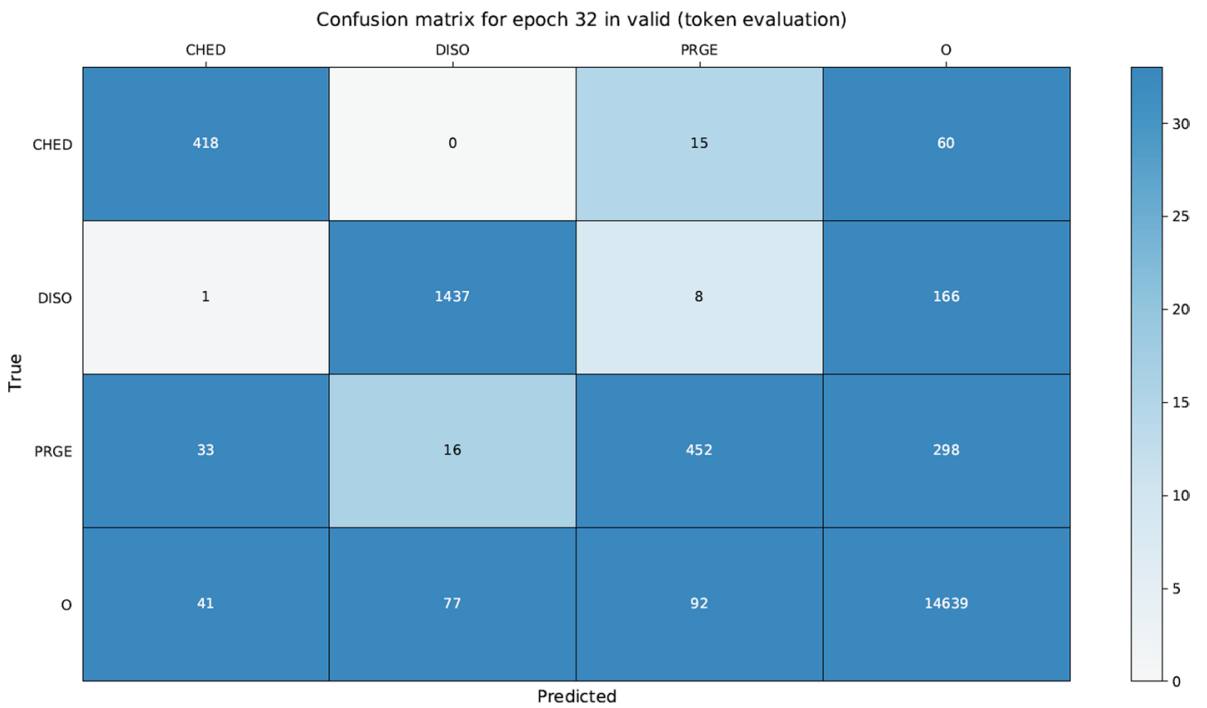

We found that the use of a domain-specific (biomedical) and language-specific pretrained models highly improve the NER task. In addition, to the best of our knowledge, there is no open pre-trained biomedical Spanish model for context-dependent word representations (pre-trained BERT). The base BERT model without extensions outperforms Extended NeuroNER model and other PharmaCoNER participant approaches, mainly due to its capability to deal with ambiguity problems.

We found that the text pre-processing (sentences split and tokenization) step had a significant impact on the entity offset recognition and classification mainly due out-of-vocabulary words. Additionally, we analyzed the confusion matrices for PharmaCoNER (see Table 15) and CORD-19 (see Table 16) datasets, where the leading diagonal represents correctly classified tokens (true positives and true negatives) and 
the cells above and below the leading diagonal misclassified tokens (false positives and false negatives). We can see for PhamarCoNER dataset that the greatest amount of missclassified tokens (269) occurs with the PROTEINAS type entity and in the same way for CORD-19 dataset the greatest amount of misclassified tokens (452) occurs with the PRGE (protein or gene) type entity. This can be attributed to a large number of abbreviations and short-length entities. Furthermore, on false positives and false negatives error analysis we found that: (i) separating words by the hyphen '-' caused some errors (e.g., S-100, Alfa-Feto-Proteina). (ii) Abbreviation recognition is a difficult task due to ambiguity and length, even more for very short abbreviations (1-2 letters) due to their high level of ambiguity (e.g., CK 7, sY86, sY84, SRY, ZFY, Hb). (iii) Long entities consisting of more than five tokens are hard to identify correctly (e.g., Antigeno Prostatico Especifico, Antigeno Carcino Embrionario). (iv) Misspelling entities cause errors in concept indexing (e.g., lacticodeshidrogenasa, tenecteplasa). (v) Also, words do not present in the pre-trained models' vocabulary are not recognized in entity offset recognition and classification.

Finally, entity recognition mistakes from offset detection and entity classification are propagated to the concept indexing task. There are about 10\% errors caused by offset detection and entity classification. In addition, about $10 \%$ errors are caused by the concept indexing model. About 40\% entities are abbreviations, which is difficult to find the appropriate concept from SNOMED-CT which only considers full concept name. Moreover, about $20 \%$ of entities have the same candidates in SNOMED-CT, which are not normalized entities in the shared task. This proves that shorter sentences and shorter entities are easier to process. Longer are the sentence more complex syntactic structures it carries, and tougher it is to be processed by the system.

\section{Conclusions}

In this work, we propose a system for biomedical concept detection such as chemical compounds, drugs, disorders, chemicals, genes, and proteins in clinical narrative and biomedical texts written in Spanish and English. We address the named entity recognition task as a sequence labeling task. Our deep learning approaches only use dense vector representations features instead of hand-crafted word-based features. We proved that as in other tasks such as NER, the use of dense representation of words such as word-level, character-level, and sense no-contextualized and contextualized representations are helpful for named entity recognition. Moreover, domain and language specific embedding models outperform general domain and cross-lingual models mainly due to the non-existence of vectors for words that are not found in the vocabulary. Our approaches achieved satisfactory performance with an F-score of $85.25 \%$ for Extended NeuroNER and $88.80 \%$ for SBC-BERT. Although the BERT model outperforms the Extended NeuroNER model, the BERT model is highly expensive to train in terms of time and cost. Besides, as mentioned before out of vocabulary words are not recognized in the offset and classification step. The Extended NeuroNER and BERT models are domain-independent and could be used in other fields, although generic and domainspecific pre-trained word representations are used for this work. Moreover, new pretrained Biomedical Spanish word embeddings (contextualized and no-contextualized) and concept embeddings have been generated for this work. 


Fig. 1 BRAT annotation example from PharmaCoNER corpus sentence where three entities are shown, two
of them nested entities - "calcio iónico corregido" and "calcio"

Table 1721 entity tags for BMEWO-V tag encoding on PharmaCoNER dataset where NORMALIZABLES and NO_NORMALIZABLES refer to chemical entities, PROTEINAS are proteins entities and UNCLEAR refer to tokens different from chemical or protein mentions [34]

\begin{tabular}{ll}
\hline Entity & Tags \\
\hline NORMALIZABLES & B/M/E/WN-NORMALIZABLES \\
NO_NORMALIZABLES & B/M/E/WN-NO_NORMALIZABLES \\
PROTEINAS & B/M/E/WN-PROTEINAS \\
UNCLEAR & B/M/E/WN- UNCLEAR \\
Others & 0 \\
\hline
\end{tabular}

More initiatives to foster the availability of sufficiently large clinical narrative corpora in Spanish from hospitals or regional health systems are necessary. This will allow us to train embeddings of different types such as knowledge enhanced word embeddings that combine text corpora with terminology resources. Resources similar to clinical concept embeddings (cui2vec) obtained in [30] from 20 million clinical notes and 1.7 million fulltext biomedical journal articles using UMLS could be useful as specialized biomedical embeddings. New approaches to extend the scope of embeddings such as [31] that use BERT to pre-trained contextualized embeddings models on structured diagnosis data from 28,490,650 patients EHR dataset to be used in disease prediction could be of great interest in clinical practice.

As future work, we plan to enhance the SNOMED-CT concept representations in concept indexing step. Furthermore, we plan to generate contextualized word representations integrating biomedical knowledge into our system such as SNOMED-CT or UMLS. The motivation would be to see whether contextualized word representations generated with biomedical knowledge can help to improve the results and provide a deep learning model for biomedical NER and concept indexing.

\section{Methods}

In this section, we described our NER approach. Additionally, we introduce the corpora used to generate our train from the scratch contextualized and no-contextualized word representations. Furthermore, we described our deep learning approaches. We first present a deep network with a pre-processing step, a learning transfer step, then two recurrent neural network layers and the last layer with CRF classifier and a deep learning model based on a multi-layer bidirectional transformer encoder. Finally, the datasets used for training, validating, and evaluating our deep learning models performance. 
Table 18 Tokens annotated for the sentence "instaurándose tratamiento con corticoides orales en forma de prednisona oral" in the ConLL-2003 format

\begin{tabular}{llllll}
\hline Token & Entity & Start offset & End offset & Tag & Tag \\
\hline instaurándose & Others & 950 & 963 & 0 & 0 \\
tratamiento & Others & 964 & 975 & 0 & 0 \\
con & Others & 976 & 979 & 0 & 0 \\
corticoides & NORMALIZABLES & 980 & 991 & B-NORMALIZABLES & W-NORMALIZABLES \\
orales & Others & 992 & 998 & 0 & 0 \\
en & Others & 999 & 1001 & 0 & 0 \\
forma & Others & 1002 & 1007 & 0 & 0 \\
de & Others & 1008 & 1010 & O & O \\
prednisona & NORMALIZABLES & 1011 & 1021 & B-NORMALIZABLES & W-NORMALIZABLES \\
oral & Others & 1022 & 1026 & O & O \\
\hline
\end{tabular}

This sentence has two drugs: "corticoides" and "prednisona" with B_NORMALIZABLES tag (start of entity) and W_ NORMALIZABLES tag (single entity)

Table 19 Biomedical Spanish corpus details

\begin{tabular}{lrrcrr}
\hline Collection ICorpus & \multicolumn{1}{c}{ IBECS } & \multicolumn{1}{c}{ SciELO } & MedlineNLM & MedlinePlus & \multicolumn{1}{c}{ UFAL } \\
\hline Documents & 168,198 & 161,710 & 330,928 & 1063 & 265,410 \\
Words & $23,648,768$ & $26,169,655$ & $4,710,191$ & 217,515 & $41,604,517$ \\
Unique Words & 184,936 & 159,997 & 20,942 & 5099 & 198,424 \\
\hline
\end{tabular}

\section{Named entity recognition}

In order to train our model, first texts must be preprocessed to create the input for the deep network. Sentences are split and tokenized using Spacy [32], an open-source library for advanced NLP with support for 26 languages. The output from the previous process is formatted to BRAT format [33]. BRAT is a standoff format where each line represents an annotation (such as entity, relation, event). We use the information from the BRAT format (see an example in Fig. 1) and then annotate each token in a sentence using the BMEWO-V extended tag encoding which is a contribution of authors. Table 17 shows an example of every possible tags for each entity type within the PharmaCoNER dataset. The BMEWO-V encoding allows us to capture information about the sequence of tokens in the sentence.

The BMEWO-V encoding distinguishes the B tag to indicate the start of an entity, the $M$ tag representing the continuity of an entity, the $E$ tag as the end of an entity, the $\mathrm{W}$ tag for indicating a single entity, and the $\mathrm{O}$ tag to represent other tokens that do not belong to any entity. The $\mathrm{V}$ tag allows representing overlapping entities. BMEWO-V is similar to other previous encodings [35]; however, we introduce the $\mathrm{V}$ tag to allow the representation of overlapping or nested entities which are usual phenomena in these types of texts. Additionally, we tested the BMEWO-V enconding format in previous works $[16,36]$. Finally, the BRAT format is transformed into sentences annotated in the CoNLL-2003 format [37]. This is the input for our deep learning models, as is shown in Table 18. 


\section{Corpora}

In order to generate from scratch Spanish biomedical word representations to use in this research we gathered raw biomedical Spanish text from different sources. Source corpus details are described in Table 19:

1 The Spanish Bibliographical Index in Health Sciences (IBECS) corpus [38] that collects scientific journals covering multiple fields in health sciences.

2 Scientific Electronic Library Online (SciELO) corpus [39] gathers electronic publications of complete full-text articles from scientific journals of Latin America, South Africa and Spain.

3 MedlineNLM corpus obtained from the PubMed free search engine [40].

4. The MedlinePlus corpus [41] (an online information service provided by the U.S. National Library of Medicine), consists of Health topics, Drugs and supplements, Medical Encyclopedia and Laboratory test information.

5 The UFAL corpus [42] is a collection of parallel corpora of medical and general domain texts.

All the corpora are in XML (Dublin core format) and TXT format files. XML files were processed for extract only raw text from specific XML tags such as "title" and "description" from Spanish labels, based on the Dublin Core format as shown in Fig. 2. TXT files were not processed. Raw texts from all files were compiled in a single TXT file. Texts were processed, setting all to lower, removing punctuation marks, trailing spaces and stop words and used as input to generate our word embeddings. Sentences pre-processing (split and tokenized) were made using Spacy [43], an open-source python library for advanced multi-language natural language processing.

\section{Bi-LSTM CRF model: extended NeuroNER}

Our proposal involves the adaption of a NER model named NeuroNER [20] based on deep learning to identify drug and chemical mentions. The architecture of our model consists of a first Bi-LSTM layer for character embeddings. In the second layer, we concatenate the output of the first layer with the word embeddings and sense-disambiguate embeddings for the second Bi-LSTM layer. Finally, the last layer uses a CRF to obtain the most suitable labels for each token. An overview of the system architecture can be seen in Fig. 3.

To facilitate our model training, we first perform a learning transfer step. Learning transfer aims to perform a task on a dataset using knowledge learned from a previous dataset [44]. As is shown in many works, such as speech recognition [45], sentence

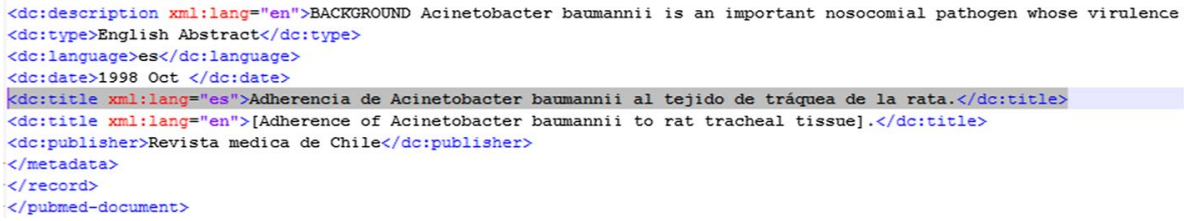

Fig. 2 Dublin core format for biomedical corpus 


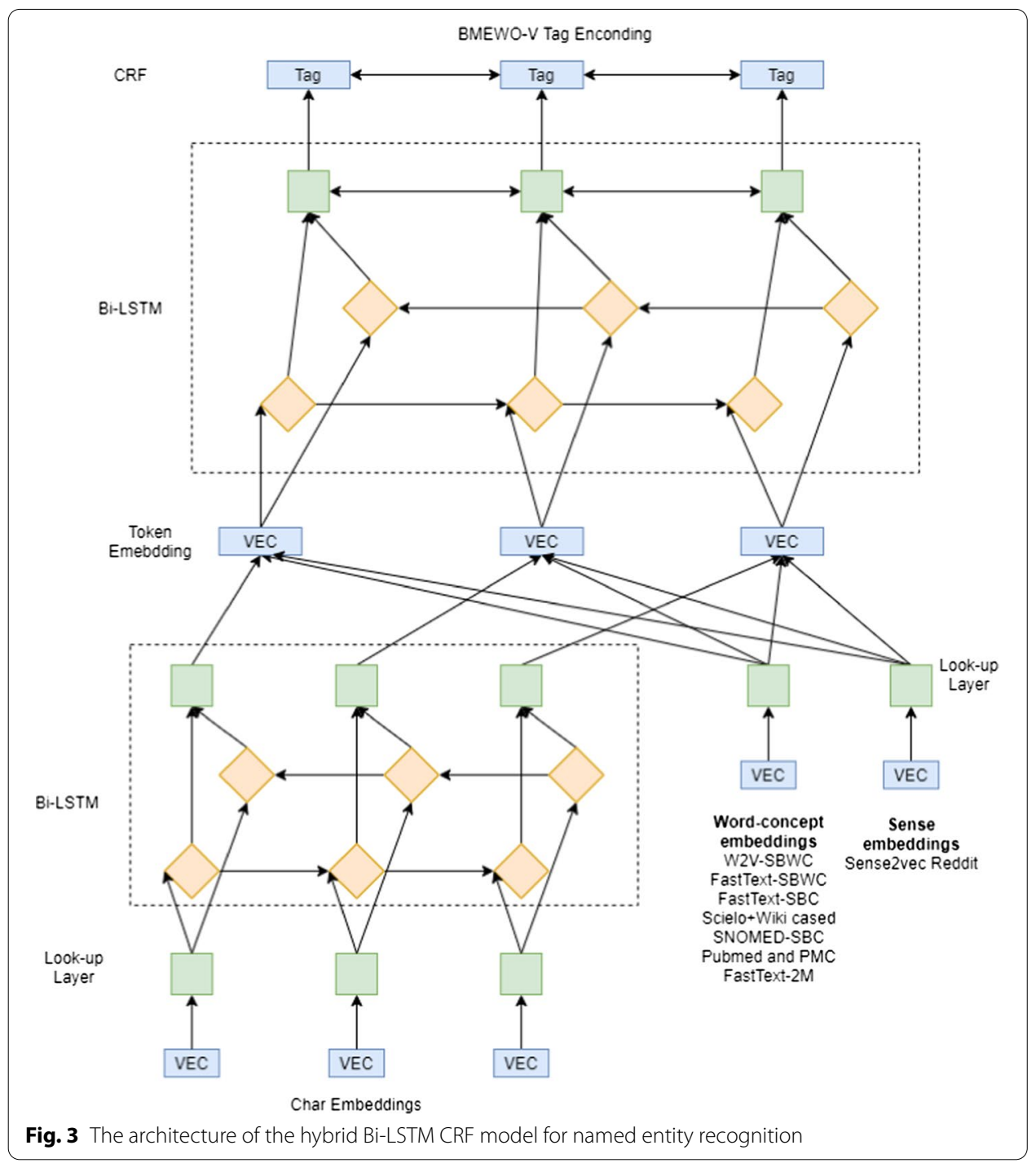

classification [46] and Named Entity Recognition [47] transfer learning improves generalization of the model, reduces training time on the target dataset, and reduces the amount of labeled data needed to obtain high performance. We propose learning transfer as input for our model using two different pre-trained embeddings models: (i) word embeddings and (ii) sense-disambiguation embeddings. Word embedding is an approach to represent words as vectors of real numbers which have gained much popularity among the NLP community because they are able to capture syntactic and semantic information among words.

Although word embedding models are able to capture syntactic and semantic information, other linguistic information such as morphological information, orthographic transcription or POS tags are not exploited in these models. According to [48], the use of character embeddings improves learning for specific domains and is useful for morphologically rich languages (as is the case of the Spanish language). For this reason, we decided to consider the character embedding representation in our system to obtain 
morphological and orthographic information from words. We used a 25 features vector to represent each character. In this way, tokens in sentences are represented by their corresponding character embeddings, which are the input for our Bi-LSTM network.

In this work, we used various Spanish and English pre-trained embedding models. The Spanish Billion Words Corpora (SBWC) [21] (W2V-SBWC), which is a pre-trained word embeddings model trained on different general domain text corpora written in Spanish (such Ancora Corpus [49] and Wikipedia) using the word2vec [50] implementation. The FastText-SBWC pre-trained word embeddings model was trained on the SBWC using the FastText implementation. The Scielo+Wiki cased [51] pre-trained word embeddings model trained on biomedical domain texts from Scielo and Wikipedia using the FastText implementation. We also integrate the sense2vec [22] model, which provides multiple dense vector representations for each word based on the sense of the word. This model is able to analyze the context of a word based on the lexical and grammatical properties of words and then assigns its more adequate vector. Each word in this model is paired with its corresponding Part-of-Speech (PoS) tag. Sense2vec use the Polyglot Part-of-Speech tagger from Al-Rfou more details in [22]. We used the Reddit Vector, a pre-trained model of sense-disambiguation representation vectors presented by [22]. This model was trained on a collection of general domain comments published on Reddit (corresponding to the year 2015) written in Spanish and English. The FastText2M [52] pre-trained English word embedding model trained with subword information on Common Crawl using the FastText implementation. Finally, the PubMed and PMC [53] pre-trained English word embedding model, trained on a combination of PubMed abstracts and full-text documents from the PMC using the word2vec skip-gram model implementation.

Furthermore, we used the FastText [54] implementation to train our own word embeddings using the Spanish Biomedical Corpora (SBC) described in section Corpora (FastText-SBC). Moreover, we trained a concept embedding model replacing biomedical concepts in the SBC with their unique SNOMED-CT Spanish Edition identifier (SNOMED-SBC). First, we applied a lowercase conversion, then we replace abbreviations with their corresponding full concept name using the Spanish Medical Abbreviation DataBase (AbreMES-DB) [27] and the SEDOM Medical Abbreviation Dictionary [28] for normalizing biomedical entities. We used the PyMedTermino library [55] for concept indexing. We proposed two dictionary-based approaches. A full-text search with the Levenshtein distance algorithm [29] was applied in a first instance for concept indexing and fuzzy search with threshold using FuzzyDict implementation [14] as a second approach for concepts not found by partial matching. The FastText model uses a combination of various subcomponents to produce high-quality embeddings. It uses a standard CBOW or skip-gram models, with position-dependent weighting, phrase representations, and sub-word information in a combined manner. The training parameters for each model are shown in Table 20. Our pre-trained models can be found in Github [56] with the corpora sources, text pre-processing, and training information.

The embedding models and their parameters are summarized in Table 21. 
Table 20 Training parameters for embeddings models built in this work

\begin{tabular}{lll}
\hline ParameterlModel & FastText-SBC & SNOMED-SBC \\
\hline Number of negatives sampled & 20 & 20 \\
Sampling threshold & $6 e^{-5}$ & $6 e^{-5}$ \\
Minimum number of word occurrences & 10 & 10 \\
Minimum length of character n-gram & 3 & 3 \\
Maximum length of character n-gram & 6 & 6 \\
Size of word vectors & 300 & 300 \\
Epochs & 10 & 10 \\
Processor & 4 Intel Xeon 2.00 GHz, 8 Cores, 16 & 4 Intel Xeon 2.00 GHz, 8 \\
& Logical Processors & Cores, 16 Logical Proces- \\
RAM & & sors \\
Corpus size & $32 \mathrm{~GB}$ & $32 \mathrm{~GB}$ \\
Training time & $1 \mathrm{~GB}$ & $1 \mathrm{~GB}$ \\
\hline
\end{tabular}

Table 21 Embedding models details

\begin{tabular}{|c|c|c|c|c|c|c|c|c|}
\hline $\begin{array}{l}\text { Embedding } \\
\text { model }\end{array}$ & Language & Domain & Type & $\begin{array}{l}\text { Corpus } \\
\text { size }\end{array}$ & $\begin{array}{l}\text { Vocab } \\
\text { size }\end{array}$ & $\begin{array}{l}\text { Array } \\
\text { size }\end{array}$ & Algorithm & Property \\
\hline W2V-SBWC & Spanish & General & Word & 1.5 billion & $68 k$ & 300 & $\begin{array}{l}\text { Word2Vec } \\
\text { Skip-gram } \\
\text { BOW }\end{array}$ & Pre-trained \\
\hline $\begin{array}{l}\text { FastText- } \\
\text { SBWC }\end{array}$ & Spanish & General & Word & 1.5 billion & $81.2 \mathrm{k}$ & 300 & $\begin{array}{l}\text { FastText } \\
\text { Skip-gram } \\
\text { BOW }\end{array}$ & Pre-trained \\
\hline FastText-SBC & Spanish & $\begin{array}{l}\text { Specific } \\
\text { (Biomedi- } \\
\text { cal) }\end{array}$ & Word & $\begin{array}{l}600 \text { bil- } \\
\text { lion }\end{array}$ & $91.7 \mathrm{k}$ & 300 & $\begin{array}{l}\text { FastText } \\
\text { Skip-gram } \\
\text { BOW }\end{array}$ & Own \\
\hline $\begin{array}{l}\text { Scielo+Wiki } \\
\text { cased }\end{array}$ & Spanish & $\begin{array}{l}\text { Specific } \\
\text { (Biomedi- } \\
\text { cal) }\end{array}$ & Word & & $50 k$ & 300 & $\begin{array}{l}\text { FastText } \\
\text { Skip-gram } \\
\text { BOW }\end{array}$ & Pre-trained \\
\hline $\begin{array}{l}\text { SNOMED- } \\
\text { SBC }\end{array}$ & Spanish & $\begin{array}{l}\text { Specific } \\
\text { (Biomedi- } \\
\text { cal) }\end{array}$ & Concept & $\begin{array}{l}600 \text { bil- } \\
\text { lion }\end{array}$ & $88.1 \mathrm{k}$ & 300 & $\begin{array}{l}\text { FastText } \\
\text { Skip-gram } \\
\text { BOW }\end{array}$ & Own \\
\hline $\begin{array}{l}\text { Pubmed and } \\
\text { PMC }\end{array}$ & English & $\begin{array}{l}\text { Specific } \\
\text { (Biomedi- } \\
\text { cal) }\end{array}$ & Word & 2 billion & $400 k$ & 300 & $\begin{array}{l}\text { Word2Vec } \\
\text { Skip-gram } \\
\text { BOW }\end{array}$ & Pre-trained \\
\hline FastText-2M & English & General & Word & $\begin{array}{l}600 \text { bil- } \\
\text { lion }\end{array}$ & 2 million & 300 & $\begin{array}{l}\text { FastText } \\
\text { Skip-gram } \\
\text { BOW }\end{array}$ & Pre-trained \\
\hline $\begin{array}{l}\text { Sense2vec } \\
\text { Reddit }\end{array}$ & $\begin{array}{l}\text { English/ } \\
\text { Spanish }\end{array}$ & General & Sense & 2 billion & $120 k$ & 128 & Sense2Vec & Pre-trained \\
\hline
\end{tabular}

\section{Multi-layer bidirectional transformer encoder: BERT}

The use of word representations from pre-trained unsupervised methods is a crucial step in NER pipelines. Previous models such as Word2Vec [50], Glove [57], and FastText [54] focused on context-independent word representations or word embeddings. However, in the last few years models focused on learning context-dependent word representations, such as ELMo [58], CoVe [59], and the state-of-the-art BERT model [60], and then fine-tune these pre-trained models on downstream tasks. BERT is a context-dependent word representation model that is based on a masked language 
Table 22 Contextualized word models details

\begin{tabular}{lllll}
\hline Detail & SBC-BERT & $\begin{array}{l}\text { Bert-base- } \\
\text { multilingual-cased }\end{array}$ & BETO cased & BioBERT-Large \\
\hline Language & Spanish & 104 languages & Spanish & English \\
Domain & Biomedical & General & General & Biomedical \\
Type & Contextual Word & Contextual Word & Contextual Word & Contextual Word \\
Corpus size & 6 billion & $3300 \mathrm{M}$ & 3 billion & 21.3 billion \\
Vocab size & $200 \mathrm{k}$ & $120 \mathrm{k}$ & $31 \mathrm{k}$ & $59 \mathrm{k}$ \\
Hidden size & 768 & 768 & 1024 & 768 \\
Algorithm & BERT train & BERT train & BERT train & BERT train \\
Property & Own & Pre-trained & Pre-trained & Pre-trained \\
\hline
\end{tabular}

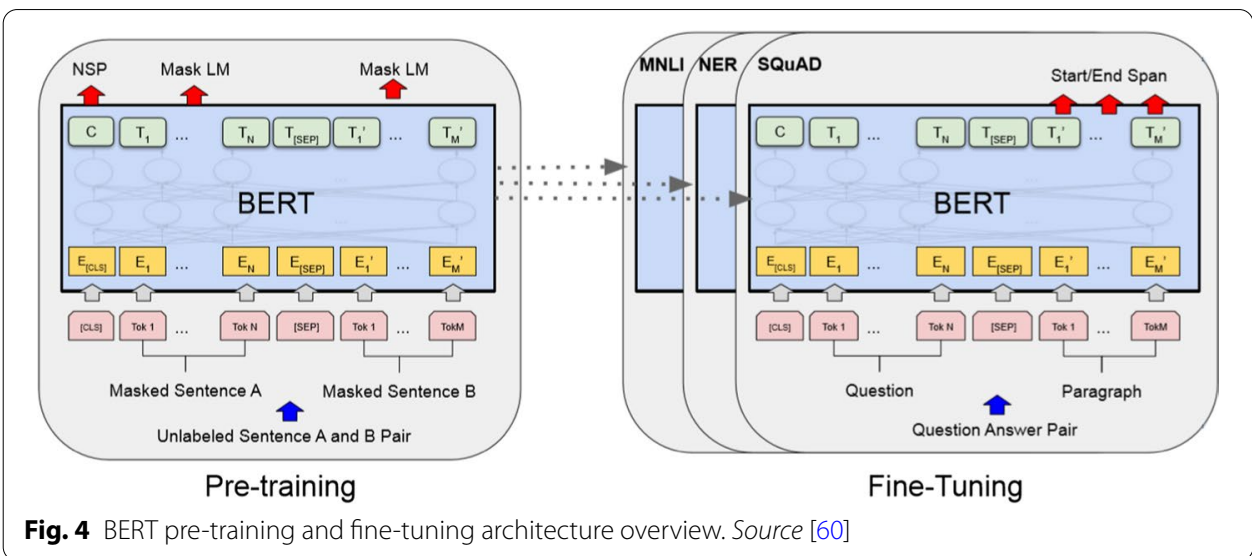

model and pre-trained using the transformer architecture [60]. BERT replaces the sequential nature of language modeling. Previous models such as RNN (LSTM and GRU) combines two unidirectional layers (i.e., Bi-LSTM), as a replacement for the sequential approach the BERT model employs a much faster attention-based approach. BERT is pre-trained in two unsupervised "artificial" tasks: (i) masked language modeling that predicts randomly masked words in a sequence, and hence can be used for learning bidirectional representations by jointly conditioning on both left and right contexts in all layers and (ii) next sentence prediction in order to train a model that understands sentence relationships. The transformer layer has two sublayers: a multi-head self-attention mechanism, and a position-wise fully connected feed-forward network, followed by a normalization layer. Even though BERT learns a lot about language through pre-training it is possible to adapt the model by adding a customized layer on top of BERT outputs and then new training is done with specific data (this phase is called fine-tuning). We refer readers [60] for a more detailed description of BERT. An overview of the BERT architecture can be seen in Fig. 4.

Due to the benefits of the BERT model, we adopted the multilingual cased [60], the BETO [61] and the Biomedical language representation (BioBERT-Large) [62] pretrained BERT models. Moreover, we trained from the scratch a Biomedical Spanish model (SBC-BERT) with 12 transformer layers (12-layer, 768-hidden, 12-heads, 110Mparameters) and a SoftMax output layer to perform the NER task. First, we replace 
Table 23 PharmaCoNER subsets details

\begin{tabular}{llccc}
\hline Dataset & Subset & Documents & Sentences & Entities \\
\hline PharmaCoNER & Train & 500 & 8036 & 3822 \\
& Valid & 250 & 3759 & 1926 \\
& Test & 3751 & 62,000 & \\
\hline
\end{tabular}

Table 24 CORD-19 subsets details

\begin{tabular}{lllll}
\hline Dataset & Subset & Documents & Sentences & Entities \\
\hline CORD-19 & Train & 8030 & 4015 & 7375 \\
& Valid & 2032 & 1016 & 1802 \\
& Test & 2776 & 1388 & 2647 \\
\hline
\end{tabular}

the WordPiece tokenizer with the SentencePiece implementation [63] and the Spacy [32] tokenizer for sentence and subword segmentation. We train with a batch size of 128 sequences for 1,000,000 steps, which is approximately 40 epochs over the 4 million word corpus. We use Adam with learning rate of $1 \mathrm{e}^{-4}$. We use a dropout probability of 0.15 on all layers and a gelu activation function. Training of SBC-BERT was performed on 1 Cloud TPU, 8vCPUs Intel(R) Xeon(R) CPU @ 2.30 GHz and 16 GB memory. Details of train and pre-trained models can be seen in Table 23.

\section{Datasets}

We evaluate our deep learning approaches on the PharmaCoNER and the COVID-19 Open Research Dataset (CORD-19) datasets. The PharmacoNER dataset is a manually annotated corpus of 1000 clinical cases written in Spanish and annotated with mentions of chemical compounds, drugs, genes, and proteins. The dataset consists of Normalizables (4398), No Normalizables (50), Proteins (3009), and Unclear (167) labels. Further details can be found in [8].

The CORD-19 dataset consists of over 181,000 scholarly articles written in English about COVID-19, SARS-CoV-2, and related coronaviruses. The dataset is manually annotated with disorder $(18,704)$, species $(30,343)$, chemical or drugs $(11,173)$, gene and protein $(57,738)$, enzyme $(1480)$, anatomy $(10,373)$, biological process $(7765)$, molecular function (1722), cellular component (1099), pathway (517) and microRNA (690) unique entities. Further details can be found in [24]. In order to compare PharmaCoNER results with CORD-19 results we only evaluate on disorder, chemical or drugs and gene and protein entities. To the best of our knowledge, the CORD-19 dataset has not been used in any NER task or challenge. Therefore, we randomly split the dataset in training, validation and test datasets. Details about the datasets can be found in Table 24.

Abbreviations

NER: Namede Entity Recogntion; Bi-LSTM: Bidirectional Long Short-Term Memory; CRF: Conditional Random Field; UMLS: Unified Medical Lanquage System; PoS: Part of Speech; SBWC: Spanish Billion Word Corpus; SBC: Spanish Biomedical Corpus; DDI: Drug Drug Interaction; SVM: Support Vector Machine; BERT: Bidirectional Encoder Representations from Transformers. 


\section{Acknowledgements}

We thank to PharmaCoNER challenge organizers, who provided publicly available training and testing datasets.

\section{About This Supplement}

This article has been published as part of BMC Bioinformatics Volume 22, Supplement 1 2021: Recent Progresses with BioNLP Open Shared Tasks-Part 2. The full contents of the supplement are available at https://bmcbioinformatics.biome dcentral.com/articles/supplements/volume-22-supplement-1.

\section{Author's contributions}

PM and RMR-Z participated in the design of the corpus and in the creation of the contextualized and no contextualized word representations. RMR-Z participated in the deep learning models implementation and the evaluation using the different word representations models. PM provided overall direction, and contributed to the writing of the manuscript. All authors read and approved the final manuscript.

\section{Funding}

This work was partially supported by the Research Program of the Ministry of Economy and Competitiveness - Government of Spain, (DeepEMR project TIN2017-87548-C2-1-R).

\section{Availability of data and materials}

The PharmaCoNER and CORD-19 datasets that support the findings of this study are available in: PharmaCoNER Datasets, https://temu.bsc.es/pharmaconer/index.php/datasets/ CORD-19 Data, https://www.kaggle.com/allen-institute-for-ai/CORD-19-research-challenge Pre-trained word and concept representations are available in:

Pubmed and PMC - Biomedical natural language processing, http://evexdb.org/pmresources/vec-space-models/ FastText-SBWC and Fastext 2M - English word vectors, https://fasttext.cc/docs/en/english-vectors.html W2V-SBWC - Spanish Billion Word Corpus and Embeddings, https://crscardellino.github.io/SBWCE/ Scielo+Wiki cased - FastText Spanish Medical Embeddings, https://doi.org/10.5281/zenodo.2542721 Sense2vec Reddit, https://pypi.org/project/sense2vec/

Bert-base-multilingual-cased - Whole Word Masking Models, https://github.com/google-research/bert BETO cased - BETO: Spanish BERT, https://github.com/dccuchile/beto BioBERT large, https://github.com/dmis-lab/biobert FastText-SBC, SNOMED-SBC, SBC-BERT trained from scratch embeddings models, https://github.com/rmriveraz/Pharm aCoNER

\section{Declarations}

\section{Consent to publish}

Not applicable.

\section{Competing interests}

The authors declare that they have no competing interests.

Received: 27 May 2021 Accepted: 9 June 2021

Published online: 17 December 2021

\section{References}

1. Bodenreider O. The Unified Medical Language System (UMLS): integrating biomedical terminology. Nucleic Acids Res. 2004;32(suppl 1):267-70. https://doi.org/10.1093/nar/gkh061.

2. Aronson A, Lang F-M. An overview of metamap: historical perspective and recent advances. J Am Med Inform Assoc JAMIA. 2010;17:229-36. https://doi.org/10.1136/jamia.2009.002733.

3. Lafferty JD, McCallum A, Pereira FCN. Conditional random fields: Probabilistic models for segmenting and labeling sequence data. In: Proceedings of the eighteenth international conference on machine learning. ICML '01. San Francisco: Morgan Kaufmann Publishers Inc.; 2001. p. 282-9. http://dl.acm.org/citation.cfm?id=645530.655813.

4. Segura-Bedmar I, Martinez P, Sanchez-Cisneros D. The 1st ddiextraction-2011 challenge task: extraction of drugdrug interactions from biomedical texts, vol. 2011; 2011. p. 1-9.

5. Segura-Bedmar I, Martínez P, Herrero-Zazo M. Lessons learnt from the ddiextraction-2013 shared task. J Biomed Inform. 2014;51:152-64. https://doi.org/10.1016/j.jbi.2014.05.007.

6. Pilevar MT, Camacho-collados J. Embeddings in natural language processing: theory and advances in vector representation of meaning. Technical report; 2020

7. Kalyan KS, Sangeetha S. SECNLP: a survey of embeddings in clinical natural language processing. J Biomed Inform. 2020;101:103323. https://doi.org/10.1016/j.jbi.2019.103323.

8. Gonzalez-Agirre A, Marimon M, Intxaurrondo A, Rabal O, Villegas M, Krallinger M. Pharmaconer: Pharmacological substances, compounds and proteins named entity recognition track. In: Proceedings of the BioNLP Open Shared Tasks (BioNLP-OST). Hong Kong: Association for Computational Linguistics; 2019. p. 1.

9. Soares F, Villegas M, Gonzalez-Agirre A, Krallinger M, Armengol-Estapé J. Medical word embeddings for Spanish: development and evaluation. In: Proceedings of the 2 nd clinical natural language processing workshop. Minneapolis: Association for Computational Linguistics; 2019. p. 124-33. https://www.aclweb.org/anthology/W19-1916.

10. Xiong Y, Shen Y, Huang Y, Chen S, Tang B, Wang X, Chen Q, Yan J, Zhou Y. A deep learning-based system for PharmaCoNER. In: Proceedings of The 5th workshop on BioNLP Open Shared Tasks. Hong Kong: Association for 
Computational Linguistics; 2019. p. 33-7. https://doi.org/10.18653/v1/D19-5706. https://www.aclweb.org/antho logy/D19-5706.

11. Stoeckel M, Hemati W, Mehler A. When specialization helps: using pooled contextualized embeddings to detect chemical and biomedical entities in Spanish. In: Proceedings of The 5th Workshop on BioNLP Open Shared Tasks. Hong Kong: Association for Computational Linguistics; 2019. p. 11-15. https://doi.org/10.18653/v1/D19-5702. https://www.aclweb.org/anthology/D19-5702.

12. León FS, Ledesma AG. Annotating and normalizing biomedical NEs with limited knowledge. 2019;1912:09152.

13. De Silva TS, MacDonald D, Paterson G, Sikdar KC, Cochrane B. Systematized nomenclature of medicine clinical terms (SNOMED CT) to represent computed tomography procedures. Comput Methods Prog Biomed. 2011;101(3):324-9. https://doi.org/10.1016/j.cmpb.2011.01.002.

14. Hemati W, Mehler A. LSTMVOTER: chemical named entity recognition using a conglomerate of sequence labeling tools. J Cheminform. 2019;11(1):3. https://doi.org/10.1186/s13321-018-0327-2.

15. Pérez-Pérez M, Rabal O, Pérez-Rodríguez G, Vazquez M, Fdez-Riverola F, Oyarzábal J, Valencia A, Lourenço A, Krallinger M. Evaluation of chemical and gene/protein entity recognition systems at biocreative v.5: the cemp and gpro patents tracks. 2017.

16. Suárez-Paniagua V, Zavala RMR, Segura-Bedmar I, Martínez P. A two-stage deep learning approach for extracting entities and relationships from medical texts. J Biomed Inform. 2019;99:103285. https://doi.org/10.1016/j.jbi.2019. 103285.

17. Weber L, Münchmeyer J, Rocktäschel T, Habibi M, Leser U. HUNER: improving biomedical NER with pretraining. Bioinformatics. 2019;36(1):295-302. https://doi.org/10.1093/bioinformatics/btz528.

18. Bada M, Eckert M, Evans D, Garcia K, Shipley K, Sitnikov D, Baumgartner W Jr, Cohen K, Verspoor K, Blake J, Hunter L. Concept annotation in the craft corpus. BMC Bioinform. 2012;13:161. https://doi.org/10.1186/1471-2105-13-161.

19. Armengol-Estapé J, Soares F, Marimon M, Krallinger M. Pharmaconer tagger: a deep learning-based tool for automatically finding chemicals and drugs in Spanish medical texts. Genomics Inform. 2019;17(2):15. https://doi.org/10. 5808/GI.2019.17.2.e15.

20. Dernoncourt F, Lee JY, Szolovits P. NeuroNER: an easy-to-use program for named-entity recognition based on neural networks. In: Proceedings of the 2017 conference on empirical methods in natural language processing: system demonstrations. Copenhagen: Association for Computational Linguistics; 2017. p. 97-102. https://doi.org/10.18653/ v1/D17-2017. https://www.aclweb.org/anthology/D17-2017.

21. Cardellino C. Spanish billion words corpus and embeddings. http://crscardellino.me/SBWCE/ (2016).

22. Trask A, Michalak P, Liu J. sense2vec: a fast and accurate method for word sense disambiguation in neural word embeddings. CoRR abs/1511.06388. arXiv:1511.06388 (2015)

23. PharmaCoNER Evaluation. https://temu.bsc.es/pharmaconer/index.php/evaluation/. Accessed 12 April 2021.

24. Lu Wang L, Lo K, Chandrasekhar Y, Reas R, Yang J, Eide D, Funk K, Kinney R, Liu Z, Merrill W, Mooney P, Murdick D, Rishi D, Sheehan J, Shen Z, Stilson B, Wade AD, Wang K, Wilhelm C, Xie B, Raymond D, Weld DS, Etzioni O, Kohlmeier S. CORD-19: the covid-19 open research dataset. arXiv. arXiv:2004.10706 (2020).

25. Gonzalez-Agirre A, Marimon M, Intxaurrondo A, Rabal O, Villegas M, Krallinger M. PharmaCoNER: pharmacological substances, compounds and proteins named entity recognition track. In: Proceedings of The 5th workshop on BioNLP open shared tasks. Hong Kong: Association for Computational Linguistics; 2019. p. 1-10. https://doi.org/10. 18653/v1/D19-5701. https://www.aclweb.org/anthology/D19-5701.

26. Sun C, Yang Z. Transfer learning in biomedical named entity recognition: an evaluation of BERT in the PharmaCoNER task. In: Proceedings of The 5th workshop on BioNLP open shared tasks. Hong Kong: Association for Computational Linguistics; 2019. p. 100-4. https://doi.org/10.18653/v1/D19-5715. https://www.aclweb.org/anthology/D19-5715.

27. ZENODO AbreMES-DB. https://zenodo.org/record/2207130\#.XvxA7ChKg2x. Accessed 12 April 2021.

28. Diccionario de Siglas Medicas. http://www.sedom.es/diccionario/. Accessed 12 April 2021.

29. Miller FP, Vandome AF, McBrewster J. Levenshtein distance: information theory, computer science, string (computer science), string metric, damerau? Levenshtein distance, spell checker, hamming distance. Alpha Press; 2009.

30. Beam AL, Kompa B, Schmaltz A, Fried I, Weber G, Palmer NP, Shi X, Cai T, Kohane IS. Clinical concept embeddings learned from massive sources of multimodal medical data. 2018;1804:01486.

31. Rasmy L, Xiang Y, Xie Z, Tao C, Zhi D. Med-BERT: pre-trained contextualized embeddings on large-scale structured electronic health records for disease prediction. arXiv:2005.12833 (2020).

32. Explosion Al: spaCy - Industrial-strength Natural Language Processing in Python. https://spacy.io/ Accessed 12 April 2021.

33. Stenetorp P, Pyysalo S, Topić G, Ohta T, Ananiadou S, Tsujii Jl. BRAT: a web-based tool for NLP-assisted text annotation. Technical report. https://dl.acm.org/citation.cfm?id=2380942 (2012).

34. Farkas R, Vincze V, Móra G, Csirik J, Szarvas G. The CoNLL-2010 shared task: learning to detect hedges and their scope in natural language text. Technical Report July. http://www.aclweb.org/anthology/W10-3001 (2010).

35. Borthwick A, Sterling J, Agichtein E, Grishman R. Exploiting diverse knowledge sources via maximum entropy in named entity recognition. In: Sixth workshop on very large corpora. https://www.aclweb.org/anthology/W98-1118 (1998).

36. Rivera Zavala R, Martinez P. The impact of pretrained language models on negation and speculation detection in cross-lingual medical text: comparative study. JMIR Med Inform. 2020;8(12):18953. https://doi.org/10.2196/18953.

37. Sang EFTK, De Meulder F. Introduction to the conll-2003 shared task: Language-independent named entity recognition. In: Proceedings of the seventh conference on natural language learning at HLT-NAACL 2003. CONLL '03, vol. 4. Association for Computational Linguistics; 2003. p. 142-7 https://doi.org/10.3115/1119176.1119195.

38. The Spanish bibliographical index in health science. http://ibecs.isciii.es. Accessed 12 April 2021.

39. Scientific electronic library online. https://scielo.org/es/. Accessed 12 April 2021.

40. National library of medicine. https://www.ncbi.nlm.nih.gov/pubmed. Accessed 12 April 2021.

41. MedlinePlus. https://medlineplus.gov/. Accessed 12 April 2021.

42. UFAL medical corpus. https://ufal.mff.cuni.cz/ufal_medical_corpus. Accessed 12 April 2021.

43. Industrial-I. https://spacy.io/. Accessed 12 April 2021. 
44. Giorgi JM, Bader GD. Transfer learning for biomedical named entity recognition with neural networks. Bioinformatics (Oxford, England). 2018;34(23):4087-94. https://doi.org/10.1093/bioinformatics/bty449.

45. Wang D, Zheng TF. Transfer learning for speech and language processing. CoRR abs/1511.06066. arXiv:1511.06066 (2015).

46. Mou L, Meng Z, Yan R, Li G, Xu Y, Zhang L, Jin Z. How transferable are neural networks in NLP applications? CoRR abs/1603.06111. arXiv:1603.06111 (2016).

47. Lee JY, Dernoncourt F, Szolovits P. Transfer learning for named-entity recognition with neural networks. In: 11th International conference on language resources and evaluation, LREC 2018. p. 4470-3. arXiv:1705.06273 (2019).

48. Ling W, Dyer C, Black AW, Trancoso I, Fermandez R, Amir S, Marujo L, Luis T. Finding function in form: compositional character models for open vocabulary word representation. In: Proceedings of the 2015 conference on empirical methods in natural language processing. Lisbon: Association for Computational Linguistics; 2015. p. 1520-30. https://doi.org/10.18653/v1/D15-1176.

49. Martí MA, Taulé M, Bertran M, Márquez L. AnCora: multilingual and multilevel annotated corpora. http://clic.ub.edu/ ancora/ancora-corpus.pdf (2007).

50. Mikolov T, Sutskever I, Chen K, Corrado G, Dean J. Distributed representations of words and phrases and their compositionality. CoRR abs/1310.4546. arXiv:1310.4546 (2013).

51. Armengol-Estapé J, Soares F, Marimon M, Krallinger M. PharmacoNER Tagge: a deep learning-based tool for automatically finding chemicals and drugs in Spanish medical texts. Genomics Inform. 2019;17(2):15. https://doi.org/10. 5808/GI.2019.17.2.e15.

52. Mikolov T, Grave E, Bojanowski P, Puhrsch C, Joulin A. Advances in pre-training distributed word representations. In: Proceedings of the international conference on language resources and evaluation (LREC 2018); 2018.

53. Pyysalo S, Ginter F, Moen H, Salakoski T, Ananiadou S. Distributional semantics resources for biomedical text processing. Technical report. https://github.com/spyysalo/nxml2txt (2013).

54. Bojanowski P, Grave E, Joulin A, Mikolov T. Enriching word vectors with subword information. CoRR abs/1607.04606. arXiv:1607.04606 (2016)

55. Lamy JB, Venot A, Duclos C. PyMedTermino: an open-source generic API for advanced terminology services. Stud Health Technol Inform. 2015;210:924-8. https://doi.org/10.3233/978-1-61499-512-8-924.

56. Zavala RMR. GitHub-rmriveraz/PharmaCoNER: Biomedical Spanish Word and Concept embeddings-pretrained models. https://github.com/rmriveraz/PharmaCoNER. Accessed 12 April 2021.

57. Pennington J, Socher R, Manning C. Glove: global vectors for word representation. In: Proceedings of the 2014 conference on empirical methods in natural language processing (EMNLP); 2014. p. 1532-43. https://doi.org/10.3115/ v1/D14-1162. arXiv:1504.06654.

58. Peters ME, Neumann M, Iyyer M, Gardner M, Clark C, Lee K, Zettlemoyer L. Deep contextualized word representations. In: Proceedings of the Conference of the North American chapter of the association for computational linguistics: human language technologies, NAACL HLT, vol. 1. Association for Computational Linguistics (ACL); 2018. p. 2227-37. https://doi.org/10.18653/v1/n18-1202. arXiv:1802.05365.

59. McCann B, Bradbury J, Xiong C, Socher R. Learned in translation: contextualized word vectors. Technical report. arXiv:1708.00107 (2017).

60. Devlin J, Chang MW, Lee K, Toutanova K. BERT: Pre-training of deep bidirectional transformers for language understanding. Technical report. arXiv:1810.04805 (2019).

61. Cañete J, Chaperon G, Fuentes R, Pérez J. Spanish pre-trained bert model and evaluation data. In: PML4DC at ICLR 2020; 2020 (to appear)

62. Lee J, Yoon W, Kim S, Kim D, Kim S, So CH, Kang J. BioBERT: a pre-trained biomedical language representation model for biomedical text mining. Bioinformatics. 2019. https://doi.org/10.1093/bioinformatics/btz682.

63. Kudo T, Richardson J. SentencePiece: a simple and language independent subword tokenizer and detokenizer for Neural Text Processing. arXiv:1808.06226 (2018).

\section{Publisher's Note}

Springer Nature remains neutral with regard to jurisdictional claims in published maps and institutional affiliations.

Ready to submit your research? Choose BMC and benefit from:

- fast, convenient online submission

- thorough peer review by experienced researchers in your field

- rapid publication on acceptance

- support for research data, including large and complex data types

- gold Open Access which fosters wider collaboration and increased citations

- maximum visibility for your research: over 100M website views per year

At BMC, research is always in progress.

Learn more biomedcentral.com/submissions 